\title{
Mycobacterium tuberculosis infection increases the number of osteoclasts and inhibits osteoclast apoptosis by regulating TNF- $\alpha$-mediated osteoclast autophagy
}

\author{
WEI LIU*, JUAN ZHOU*, FEI NIU*, FEIFEI PU, ZHIWEI WANG, MI HUANG, \\ XIAOLONG ZHAO, LIN YANG, PENGFEI TAO, PING XIA and JING FENG \\ Department of Orthopaedics, First Hospital of Wuhan, Wuhan, Hubei 430022, P.R. China
}

Received May 24, 2019; Accepted December 11, 2019

DOI: $10.3892 /$ etm.2020.8903

\begin{abstract}
Osteoarticular tuberculosis, a chronic inflammatory disease characterized by Mycobacterium tuberculosis (M.tb) infection, has become a serious problem in China. The present study was conducted to determine the mechanism of action of tumor necrosis factor (TNF)- $\alpha$ in the pathogenesis of osteoarticular tuberculosis. The number of osteoclasts in osteoarticular tuberculosis tissue samples was detected by tartrate-resistant acid phosphatase staining. Autophagy and apoptosis of osteoclasts were detected by western blotting, reverse transcription-quantitative PCR, transmission electron microscopy and TUNEL staining. The results showed that autophagy and the number of osteoclasts increased in the lesions of patients with osteoarticular tuberculosis compared with osteoarthritis samples. Moreover, activation of osteoclast autophagy inhibited the apoptosis of osteoclasts infected with M.tb, and increased the expression level of TNF- $\alpha$. The results showed that TNF- $\alpha$ enhanced the autophagic activity of $M . t b$-infected osteoclasts and inhibited cell apoptosis. These findings indicated that M.tb infection induced osteoclast production and inhibited osteoclast apoptosis by regulating TNF- $\alpha$-mediated osteoclast autophagy, revealing a new mechanism for TNF- $\alpha$ in the pathogenesis of osteoarticular tuberculosis.
\end{abstract}

\section{Introduction}

Osteoarticular tuberculosis is a chronic inflammatory disease characterized by Mycobacterium tuberculosis (M.tb) infection

Correspondence to: Dr Jing Feng or Dr Ping Xia, Department of Orthopaedics, First Hospital of Wuhan, 215 Zhongshan Road, Wuhan, Hubei 430022, P.R. China

E-mail: fjboys80@qq.com

E-mail: xiapingfm@126.com

*Contributed equally

Key words: osteoclast, Mycobacterium tuberculosis, osteoarticular tuberculosis, autophagy, tumor necrosis factor- $\alpha$ that occurs through the respiratory tract and spreads through the blood to the bones and joints (1). This disease is characterized by enhanced bone and joint absorption and bone destruction (1). Osteoarticular tuberculosis causes limb deformity and nerve compression, greatly affecting the quality of life of patients and imposing a global health and socio-economic burden (2). Clinically, osteoarticular tuberculosis accounts for $\sim 10 \%$ of all extrapulmonary tuberculosis cases, which is second only to pleural and lymphatic tuberculosis (3). Antituberculosis drugs have been widely used since the mid-20th century to effectively control this infection (4); however, to the best of our knowledge, the pathogenesis of osteoarticular tuberculosis has not been widely studied. In recent years, drug-resistant tuberculosis, particularly multidrug-resistant tuberculosis, has emerged globally (5). Additionally, an increase in longer chemotherapy cycles and drug side effects have presented major challenges to effectively control osteoarticular tuberculosis $(6,7)$. Therefore, the pathogenic mechanism of osteoarticular tuberculosis must be determined to develop new and effective prevention and treatment strategies.

The earliest study of the pathogenesis of osteoarticular tuberculosis was reported in 1997, which suggested that heat shock protein Cpn10 in M.tb can promote chemotaxis of osteoclasts and locally infiltrate lesions, while inhibiting proliferation of osteoblast precursors and increasing bone resorption. Furthermore, histopathological analysis of samples from patients with osteoarticular tuberculosis showed that local abnormal activation of osteoclasts lead to bone destruction (8). In 2015 a study conducted using an osteoarticular tuberculosis rabbit animal model confirmed that the number of osteoclasts in the damaged spine increased, while the number of osteoblasts decreased (9). Therefore, increased numbers and activation of osteoclasts after M.tb infection are key factors leading to the pathogenesis of osteoarticular tuberculosis.

Autophagy is a basic metabolic process in cells and involves the degradation of intracellular proteins and invading pathogens by lysosomal pathways to maintain cell survival. This is the most primitive innate immune mechanism used by eukaryotic cells to clear invading pathogens $(10,11)$. In a rheumatoid arthritis model, tumor necrosis factor (TNF)- $\alpha$ caused inflammation of the synovial membrane in the joint, increased expression of the autophagy pathway molecules 
autophagy-related protein (Atg)7 and Beclin1 in osteoclasts and promoted autophagy (12). A previous study demonstrated that TNF- $\alpha$ regulates autophagy and affects osteoclast production (13). Autophagy is also an innate immune mechanism by which macrophages control M.tb infection (14). Macrophage autophagy is activated through the action of type $1 \mathrm{~T}$ helper (Th1) cytokines such as interferon- $\gamma$, leading to degradation of phagocytic M.tb via the lysosomal pathway, preventing the proliferation of $M . t b$ in macrophages and avoiding cell necrosis (15). However, whether Th1 cells and their cytokine networks activated by M.tb infection in patients with osteoarticular tuberculosis regulate and maintain the autophagy of infected osteoclasts remains to be elucidated.

In the present study, clinical specimens were evaluated and cell experiments were conducted. TNF- $\alpha$ activated autophagy and inhibited apoptosis of osteoclasts infected with M.tb, thereby maintaining cell homeostasis. The results have practical value for guiding the prevention and treatment of osteoarticular tuberculosis.

\section{Materials and methods}

Patients and samples. Patients were divided into two groups: The osteoarticular tuberculosis group and osteoarthritis (OA) group. The selection criteria for the osteoarticular tuberculosis group were as follows: Hospitalized patients from the First Hospital of Wuhan (Wuhan, China) were diagnosed as having osteoarticular tuberculosis according to their medical history, clinical symptoms, signs, imaging findings, related laboratory tests, and pathological examination. Lesions with necrotic bone and the synovium were collected as specimens, and 30 cases were included (14 males and 16 females; age range 61-70 years). The selection criteria for the OA group were as follows: Hospitalized cases from the First Hospital of Wuhan were diagnosed as OA according to their medical history, clinical symptoms, signs, imaging findings and relevant laboratory tests. Additionally, patients with surgical indications requiring joint replacement were included. Bone and synovial tissue removed during the operation were collected as specimens from a total of 25 cases (11 males and 14 females; age range 63-72 years). All 55 specimens were collected from January 2017 to December 2018. All experimental protocols were approved by the Clinical Research Ethics Committee of Wuhan First Hospital, Tongji Medical College, Huazhong University of Science and Technology. All patients or their parents provided informed consent.

Isolation and culture of human osteoclasts. The standards for healthy volunteers were as follows: Males aged 20-30 years, no metabolic bone disease according to blood and urine analysis, normal liver and kidney function, no hereditary, blood, or infectious diseases and no general infections, such as colds, within the past 1 month. Samples were collected at Wuhan First Hospital from January 2017 to December 2018. Informed consent was provided by the volunteers prior to elbow vein blood collection. Healthy male volunteers were selected, and $80-100 \mathrm{ml}$ of whole blood was collected from the venous blood. Human monocytes were separated by density gradient centrifugation. DMEM (Gibco; Thermo Fisher Scientific, Inc.) was mixed with equal volumes of heparin-anticoagulated whole blood. Diatrizoate was added to a $50 \mathrm{ml}$ centrifuge tube, and the mixture was slowly added along the tube wall to ensure a clear interface between the two samples, which were then centrifuged at $362 \mathrm{xg}$ at $4^{\circ} \mathrm{C}$ for $30 \mathrm{~min}$. The obtained mononuclear cells were placed in two 25 -mm petri dishes and cultured in $5 \% \mathrm{CO}_{2}$ at $37^{\circ} \mathrm{C}$ for $24 \mathrm{~h}$, before being washed three times with DMEM. Unattached cells were eluted, and the remaining adherent cells were considered as mononuclear cells. Confluent cells were resuspended in DMEM containing 10\% FBS (Gibco; Thermo Fisher Scientific, Inc.), $100 \mathrm{mg} / \mathrm{ml}$ streptomycin, $100 \mathrm{U} / \mathrm{ml}$ penicillin, $30 \mathrm{ng} / \mathrm{ml}$ macrophage colony-stimulating factor (PeproTech, Inc.) and $40 \mathrm{ng} / \mathrm{ml}$ receptor activator of NF- $\mathrm{KB}$ ligand (PeproTech, Inc.). Cells were then incubated at $37^{\circ} \mathrm{C}$ in a humidified atmosphere with $95 \%(\mathrm{v} / \mathrm{v})$ air and $5 \%(\mathrm{v} / \mathrm{v}) \mathrm{CO}_{2}$. The culture medium was replaced every three days for 21 days.

Tartrate-resistant acid phosphatase (TRAP) staining. Patient-derived osteoclast droplets were placed on a loading slide to prepare a cell smear. The slide was allowed to dry naturally, and TRAP (Beijing Solarbio Science \& Technology Co., Ltd.) fixative was added to fix the cells at $4^{\circ} \mathrm{C}$ for $60 \mathrm{sec}$. After washing the slides with water and slight drying, the samples were placed in TRAP incubation solution. Slides were then incubated at $37^{\circ} \mathrm{C}$ for $60 \mathrm{~min}$ in the dark, and then washed with water. Cells were stained with hematoxylin for 5 min or methyl green solution for $3 \mathrm{~min}$ at $37^{\circ} \mathrm{C}$. The samples were washed with water, dried, and examined using a light microscope at $40 \mathrm{X}$ objective.

M.tb infection and treatment. A homogenizer seal was first weighed. A pipette tip was used to scrape the bacteria (provided by Professor Fan Xionglin, School of Basic Medicine, Tongji Medical College, Huazhong University of Science and Technology) from the plate and the homogenizer was sterilized and sealed, weighing the homogenizer. The dry weight of the bacteria was calculated, and $1 \mathrm{ml}$ of complete DMEM was added to the sample. The osteoclasts were ground and the supernatant was collected by centrifugation at $362 \mathrm{x}$ g for $5 \mathrm{~min}$ at $4^{\circ} \mathrm{C}$. A total of $1 \mathrm{ml}$ supernatant was added to the autophagy gene knockout type M.tb H37Rv (M.tb H37Rv $\Delta$ eis) and wild-type M.tb H37Rv (M.tb H37Rv WT) cultured complete DMEM and then mixed well. Osteoclasts not infected with M.tb were used as a blank control group. Following infection, cells were incubated at $37^{\circ} \mathrm{C}$ for $24 \mathrm{~h}$ and osteoclasts were centrifuged at $362 \mathrm{xg}$ at $4^{\circ} \mathrm{C}$ for $5 \mathrm{~min}$ and washed twice with PBS.

For TNF- $\alpha$ treated experiments, infected cells were treated with 40 ng/ml TNF- $\alpha$ (cat. no. 1217202; Dakewe Biotech Co., Ltd.) for $24 \mathrm{~h}$ or pre-treated with $10 \mu \mathrm{M} 3$-methyladenine (3-MA) (cat. no. M9281; Sigma-Aldrich, Merch KGaA) before TNF- $\alpha$ administration.

$R N A$ extraction and reverse transcription-quantitative $P C R$ $(R T-q P C R)$. Total RNA was isolated from the harvested cells and tissues using TRIzol ${ }^{\mathrm{TM}}$ reagent (Invitrogen; Thermo Fisher Scientific, Inc.) according to the manufacturer's protocol. The RNA was denatured for $5 \mathrm{~min}$ at $70^{\circ} \mathrm{C}$ and placed on ice for 5 min. Denatured RNA was added to a mixture of MMLV-RT, MMLV-RT buffer, horseradish peroxidase (HRP) RNA-RNA interaction/RNase inhibitor and dNTPs and incubated for 
Table I. List of primers used for reverse transcription-quantitative PCR.

Primer sequence $\left(5^{\prime} \rightarrow 3^{\prime}\right)$

\begin{tabular}{|c|c|c|c|}
\hline Gene & GenBank accession & Forward & Reverse \\
\hline Human Bax & NM_003217 & CATATAACCCCGTCAACGCAG & GCAGCCGCCACAAACATAC \\
\hline Human Bcl-2 & AF089746 & GTCTTCGCTGCGGAGATCAT & CATTCCGATATACGCTGGGAC \\
\hline Human Caspase 3 & NM_004346 & CATGGAAGCGAATCAATGGACT & CTGTACCAGACCGAGATGTCA \\
\hline Human Atg7 & NM_001144912 & CAGTTTGCCCCTTTTAGTAGTGC & CCAGCCGATACTCGTTCAGC \\
\hline Human Beclin 1 & NM_017749 & CTGGTAGAAGATAAAACCCGGTG & AGGTAGAGCGTGGACTATCCG \\
\hline Human GAPDH & NM_002046 & CTGCTCCTCCTGTTCGACAGT & CCGTTGACTCCGACCTTCAC \\
\hline
\end{tabular}

Atg7, autophagy-related protein 7.

$60 \mathrm{~min}$ at $42^{\circ} \mathrm{C}$. The mixture was inactivated by heating at $95^{\circ} \mathrm{C}$ for 5 min. qPCR was performed using Power SYBR Green PCR Master Mix (Thermo Fisher Scientific, Inc.) on a 7900HT thermocycler (Applied Biosystems; Thermo Fisher Scientific, Inc.). The following thermocycling conditions were used for the PCR: Initial denaturation at $50^{\circ} \mathrm{C}$ for $2 \mathrm{~min} ; 40$ cycles of $95^{\circ} \mathrm{C}$ for $10 \mathrm{~min}, 95^{\circ} \mathrm{C}$ for $30 \mathrm{sec}$ and $60^{\circ} \mathrm{C}$ for $30 \mathrm{sec}$; and a final extension step at $60^{\circ} \mathrm{C}$ for $30 \mathrm{sec}$. A total of $1 \mu \mathrm{g} \mathrm{cDNA}$ and $0.4 \mu \mathrm{l}$ of primer were used for the qPCR, Relative gene expression levels were calculated using the $2^{-\Delta \Delta C q}$ method (16). GAPDH was used as an endogenous control to normalize the level of each mRNA. The sequences of the primers used are shown in Table I. All experiments were performed at least in triplicate.

Western blotting. Osteoclasts were rinsed 2-3 times with TBS. An appropriate volume of RIPA buffer (Biosharp) was added to plates and flasks for 3-5 min followed by incubation in an ice bath for $30 \mathrm{~min}$. The supernatant was collected as a total protein solution. Total, cytoplasmic, and mitochondrial proteins were extracted using corresponding kits (Beyotime Institute of Biotechnology). Protein concentration was determined using a BCA protein assay kit. A total of $20 \mu \mathrm{g} /$ lane extracted proteins were separated by $10 \%$ SDS-PAGE and transferred to PVDF membranes (EMD Millipore). After blocking in 5\% non-fat dried milk in TBS-Tween-20 (TBS-T) at $37^{\circ} \mathrm{C}$ for $2 \mathrm{~h}$, the blots were incubated with anti-Atg7 (1:500; cat. no. Ag27914; ProteinTech Group, Inc.), anti-Beclin1 (1:500; cat. no. 11306-1-AP; ProteinTech Group, Inc.), anti-Bax (1:1,000; cat. no. 50599-2-Ig; ProteinTech Group, Inc.), anti-Bcl-2 (1:1,000; cat. no. 10927-1-AP; ProteinTech Group, Inc.), anti-cytochrome C (1:500; cat. no. 12245-1-AP; ProteinTech Group, Inc.), anti-cleaved-caspase 3 (1:1,000; 19677-1-AP; ProteinTech Group, Inc.), anti-microtubule-associated protein 1A/1B light chain 3A (LC-3; 1:500; cat. no. 18725-1-AP; ProteinTech Group, Inc.), anti- $\beta$-actin (1:1,000; cat. no. TDY051; Beijing TDY Biotech Co., Ltd.) and anti-voltage-dependent anion channel (1:3,000; cat. no. sc-32063; Santa Cruz Biotechnology; Dallas, TX, USA), overnight at $4^{\circ} \mathrm{C}$. $\beta$-actin served as an internal control. The membranes were then washed three times with TBS-T on a bleaching shaker at room temperature for $5 \mathrm{~min}$. HRP-labeled goat anti-mouse antibody $(1: 3,000$; cat. no. BL002A; Biosharp) diluted with TBS-T was incubated with the membranes for $30 \mathrm{~min}$ at room temperature. The membranes were washed three times with TBS-T on a decolorizing shaker at room temperature for $5 \mathrm{~min}$. A total of $1 \mathrm{ml}$ Electrochemiluminescence A (ECLA) (cat. no. BL520A; Biosharp) and electrochemiluminescence B (ECLB) (cat. no. BL520A; Biosharp) reagents were mixed in a centrifuge tube. The protein side of the PVDF membrane was brought into full contact with the mixed solution. After 1-2 min, the residual liquid was removed and the membrane was incubated in the dark. Protein expression was quantified using ImageJ v1.46 (National Institutes of Health). All experiments were performed at least in triplicate.

ELISA. The level of TNF- $\alpha$ in culture supernatant was measured using ELISA kits (cat. no. E-EL-H0109c; Elabscience) according to the manufacturer's instructions. Optical density was detected by a microplate reader at a wavelength of $450 \mathrm{~nm}$.

TUNEL. DNA fragmentation was detected using an In Situ Cell Death Detection kit (Roche Diagnostics). After fixation with $4 \%$ paraformaldehyde at $37^{\circ} \mathrm{C}$ for $1 \mathrm{~h}$, the cells were incubated with $3 \% \mathrm{H}_{2} \mathrm{O}_{2}$ and $0.1 \%$ Triton $\mathrm{X}-100$ for $10 \mathrm{~min}$ and washed with PBS three times following each step. In accordance with standard protocols, the cells were stained with TUNEL inspection fluid (1:100) and DAPI at $37^{\circ} \mathrm{C}$ for $1 \mathrm{~h}$. The slides were mounted on a mounting solution containing an anti-fluorescent quencher (Fluoromont-G; cat. no. 0100-01; SouthernBiotech). Three fields of view on each slide were randomly chosen for observation with a fluorescence microscope.

Transmission electron microscopy. Medium was first decanted before cells were fixed with $2.5 \%$ glutaraldehyde (Sinopharm Chemical Reagent Co., Ltd.) at $4^{\circ} \mathrm{C}$ for $15 \mathrm{~min}$. Cells were subsequently collected by centrifugation at $362 \times \mathrm{g}$ and $37^{\circ} \mathrm{C}$ and stored at $4^{\circ} \mathrm{C}$. Cells were rinsed three times with $0.1 \mathrm{M}$ phosphate buffer (Sinopharm Chemical Reagent Co., Ltd.) for $15 \mathrm{~min}$, followed by dehydration in a 30, 50, 70, 80, 85, 90 and $100 \%$ ethanol gradient for 15-20 min in each alcohol solution. The penetrant was composed of epoxy resin (Sinopharm Chemical Reagent Co., Ltd.) and acetone (Sinopharm Chemical Reagent Co., Ltd.). The infiltrated sample was placed in an embedding plate, before addition of embedding 
A
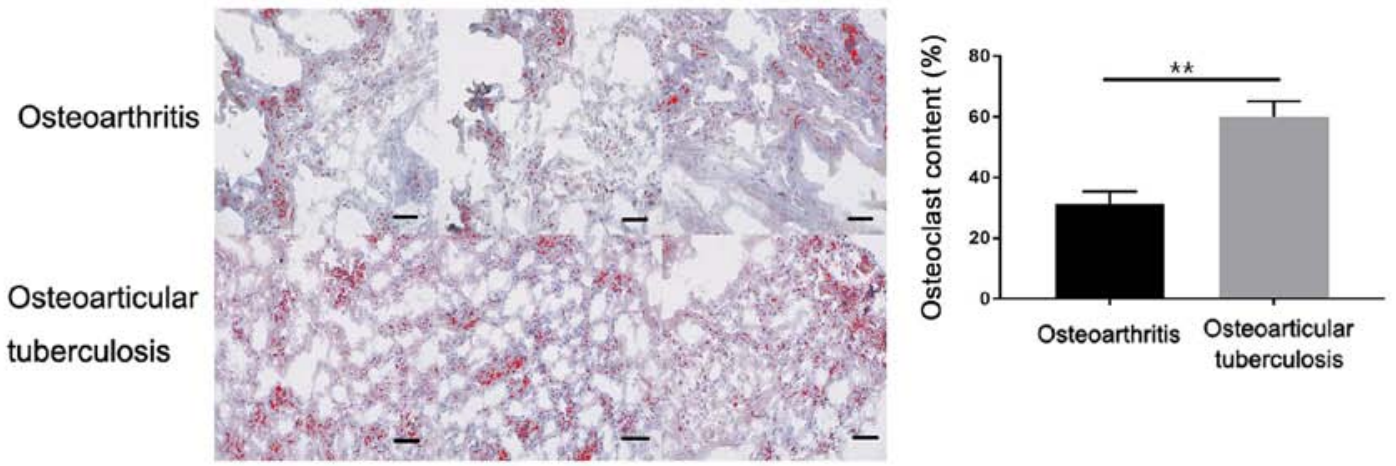

B
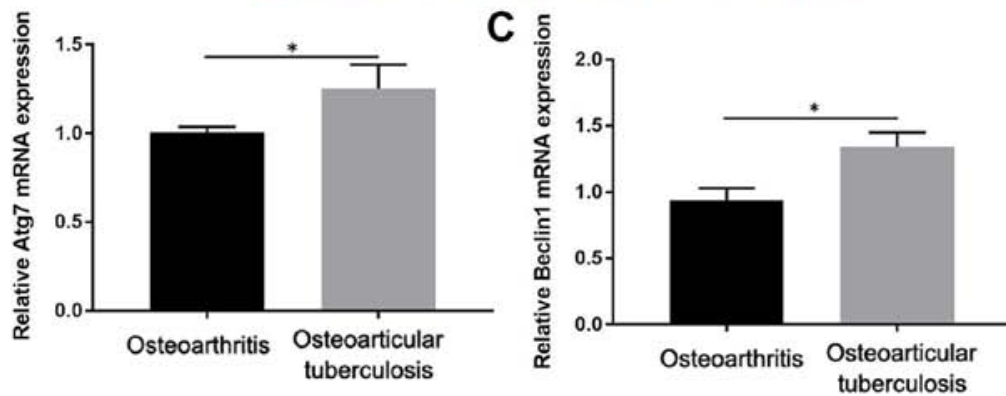

D

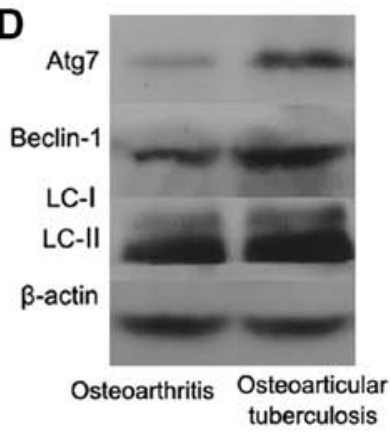

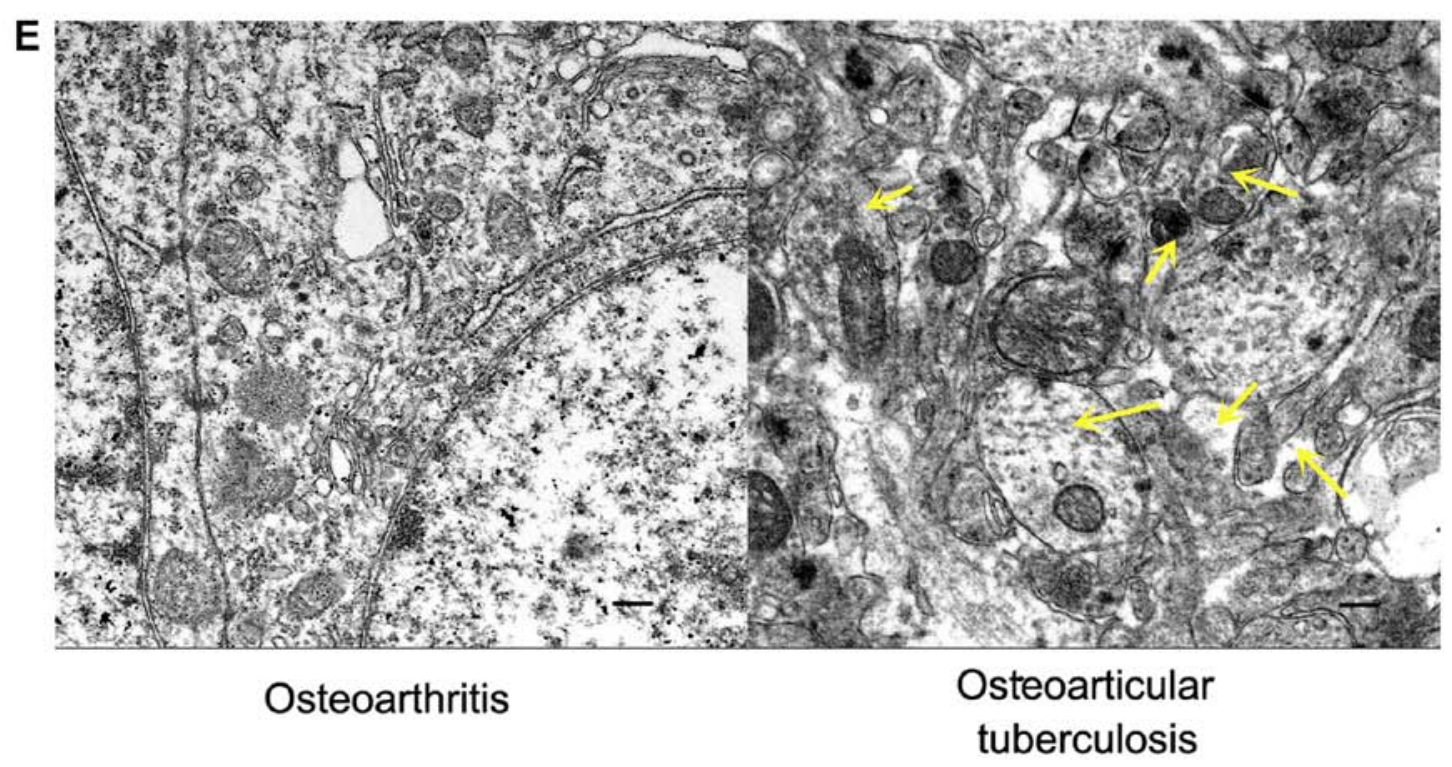

Figure 1. Autophagy is enhanced in osteoarticular tuberculosis tissues. (A) Representative images of tartrate-resistant acid phosphatase staining of pathological tissues from 3 different patients with osteoarthritis and osteoarticular tuberculosis and quality analysis of pathological tissues from osteoarthritis (25 cases) and osteoarticular tuberculosis (30 cases). Scale bar $=1 \mu \mathrm{m}$. (B) mRNA expression levels of Atg7 in osteoarthritis and osteoarticular tuberculosis pathological tissues. (C) mRNA expression levels of Beclin1 in osteoarthritis and osteoarticular tuberculosis pathological tissues. (D) Protein levels of Atg7, Beclin1 and LC3-II/I in osteoarthritis and osteoarticular tuberculosis pathological tissues. (E) Autophagosomes in osteoclasts from osteoarthritis and osteoarticular tuberculosis pathological tissues were observed by transmission electron microscopy (magnification, x5,000). Yellow arrows indicate autophagosomes. Scale bar $=1 \mu \mathrm{m}$. Data are represented as the mean $\pm \mathrm{SD} .{ }^{*} \mathrm{P}<0.05,{ }^{* *} \mathrm{P}<0.01$. Atg7, autophagy-related protein 7 ; LC 3 , microtubule-associated proteins $1 \mathrm{~A} / 1 \mathrm{~B}$ light chain $3 \mathrm{~A}$.

epoxy resin. The samples were embedded with epoxy resin (Hubei Xinkang Pharmaceutical Chemical Co., Ltd.) in a $60^{\circ} \mathrm{C}$ incubator for $48 \mathrm{~h}$. An ultramicrotome was used to slice the sections to $80-100 \mathrm{~nm}$ thickness. Uranyl acetate was added to the sections and incubated at room temperature for $15 \mathrm{~min}$. The sections were dried overnight at room temperature and observed under an electron microscope.

Statistical analysis. The results are presented as the mean \pm SD. Statistical analysis was performed using SPSS 20.0 software (IBM Corp.). Two groups were compared using an independent sample t-test, and three or more groups were compared by one-way ANOVA followed by Dunnett's multiple comparison test. $\mathrm{P}<0.05$ was considered to indicate a statistically significant difference.

\section{Results}

Autophagy is enhanced in osteoarticular tuberculosis tissues. The number of osteoclasts in 30 osteoarticular tuberculosis pathological tissues and 25 osteoarthritis pathological tissues was detected by TRAP staining. As shown in Fig. 1A, the osteoclast 

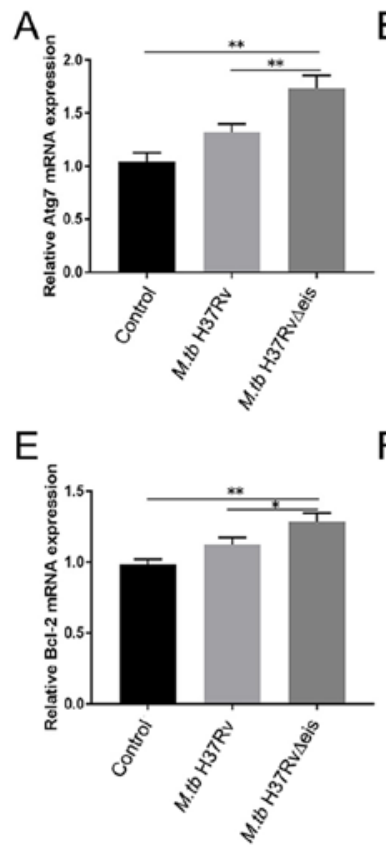

$\mathrm{F}$
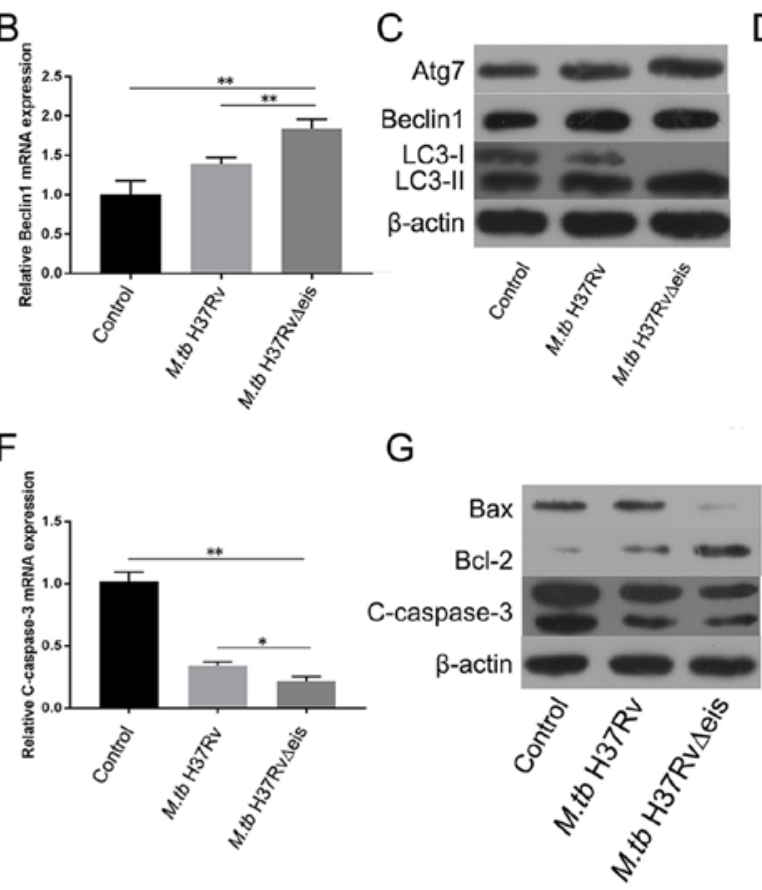

G
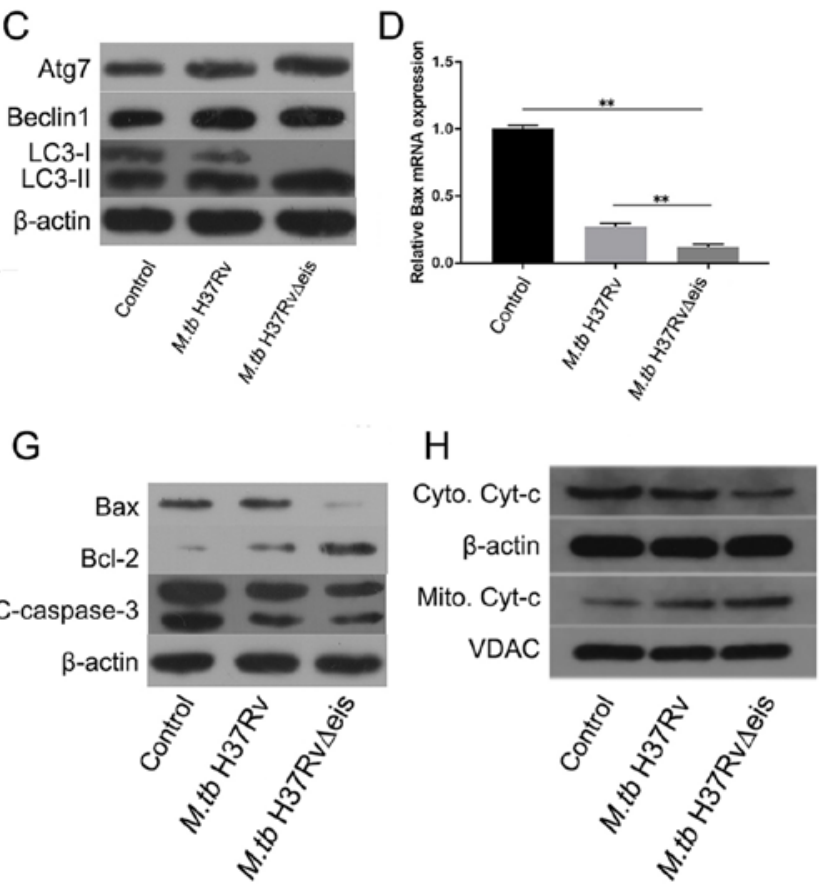

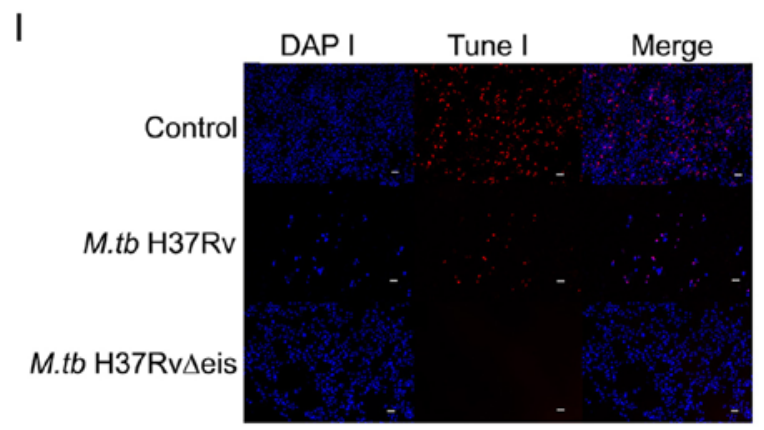

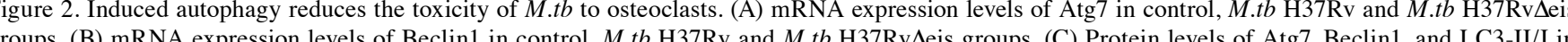
control, M.tb H37Rv and M.tb H37Rv $\Delta$ eis groups. mRNA expression levels of (D) Bax, (E) Bcl-2, and (F) cleaved-caspase 3 in control, M.tb H37Rv infection and M.tb H37Rv $\Delta$ eis infection groups. (G) Protein levels of Bax, Bcl-2 and cleaved-caspase 3 in control, M.tb $\mathrm{H} 37 \mathrm{Rv}$ and M.tb $\mathrm{H} 37 \mathrm{Rv} \Delta$ eis groups. (H) Western blotting assay of the levels of Cyt-c in cytoplasmic and mitochondrial extracts from control, M.tb H37Rv infection and M.tb H37Rv $\Delta$ eis infection groups. (I) TUNEL assay was performed in osteoclasts in control, M.tb H37Rv and M.tb H37RvAeis osteoclasts (Magnification, x200; scale bars, $50 \mu \mathrm{m}$ ). Data are presented as the mean $\pm \mathrm{SD} .{ }^{*} \mathrm{P}<0.05$ and ${ }^{* *} \mathrm{P}<0.01$. M.tb H37Rv $\Delta$ eis, inhibition of autophagy gene knockout type Mycobacterium tuberculosis $\mathrm{H} 37 \mathrm{Rv}$; M.tb H37Rv, wild type Mycobacterium tuberculosis H37Rv; Atg7, autophagy-related protein 7; LC-3, microtubule-associated proteins 1A/1B light chain 3A; Cyt-c, cytochrome-c; VDAC, voltage-dependent anion channel.

content was markedly increased in osteoarticular tuberculosis pathological tissues compared with OA samples. Autophagic activity during osteoarticular tuberculosis was subsequently investigated. RT-qPCR was performed to detect Atg7 and Beclin1 mRNA levels in osteoarticular tuberculosis tissues. The results showed that Atg7 and Beclin1 mRNA levels were significantly increased in osteoarticular tuberculosis samples compared with OA samples (Fig. 1B and C). Additionally, the expression of Atg7, Beclin1 and LC3-II/I protein were investigated by western blotting. The results showed that the protein levels of Atg7, Beclin1 and LC3-II/I were increased in osteoarticular tuberculosis pathological tissue samples compared with OA samples (Fig. 1D). Furthermore, transmission electron microscopy was conducted to observe autophagy. As shown in Fig. 1E, increased accumulation of autophagosomes was observed in osteoclasts from tuberculosis lesions, as indicated by yellow arrows. The results indicated that autophagy was enhanced in osteoarticular tuberculosis compared with OA samples.
Autophagy induction reduces the toxicity of M.tb to osteoclasts. Osteoclasts were transfected with M.tb H37RvAeis or M.tb H37Rv and mRNA expression of Atg7 and Beclin1 was investigated by RT-qPCR. As shown in Fig. 2A and B, M.tb $\mathrm{H} 37 \mathrm{Rv} \Delta \mathrm{eis}$ increased the mRNA expression levels of Atg7 and Beclin1 compared with the M.tb $\mathrm{H} 37 \mathrm{Rv}$ infection group and control group. Western blot analysis was performed to detect Atg7, Beclin1, and LC3-II/I protein levels in osteoclasts. The results showed that the expression levels of these proteins were increased in the M.tb H37Rv $\Delta$ eis infection group compared with the control group (Fig. 2C).

The role of autophagy in regulating apoptosis of osteoclasts infected with M.tb. was studied using M.tb H37Rv $\Delta$ eis- and M.tb H37Rv-infected osteoclasts. RT-qPCR was performed to detect the apoptotic markers Bax, Bcl-2, and cleaved-caspase 3 mRNA levels in osteoclasts. Bax and cleaved-caspase 3 mRNA levels were significantly reduced in the H37Rv $\Delta$ eis-infected group compared with controls and 


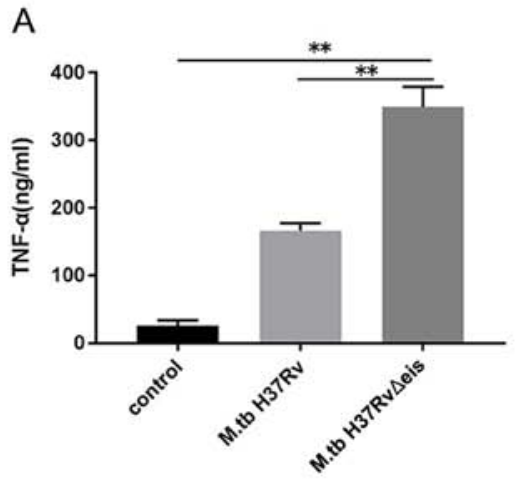

C

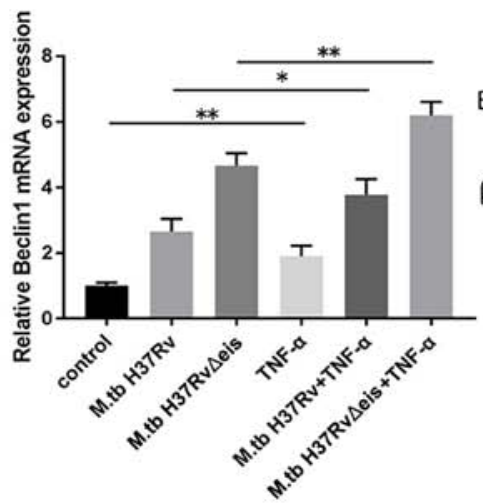

B

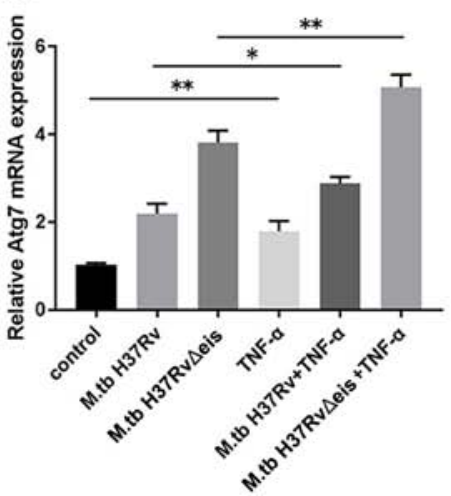

Atg7

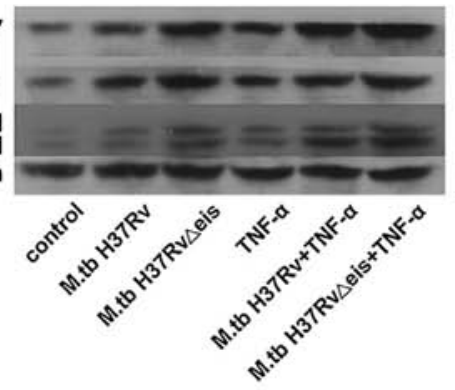

E

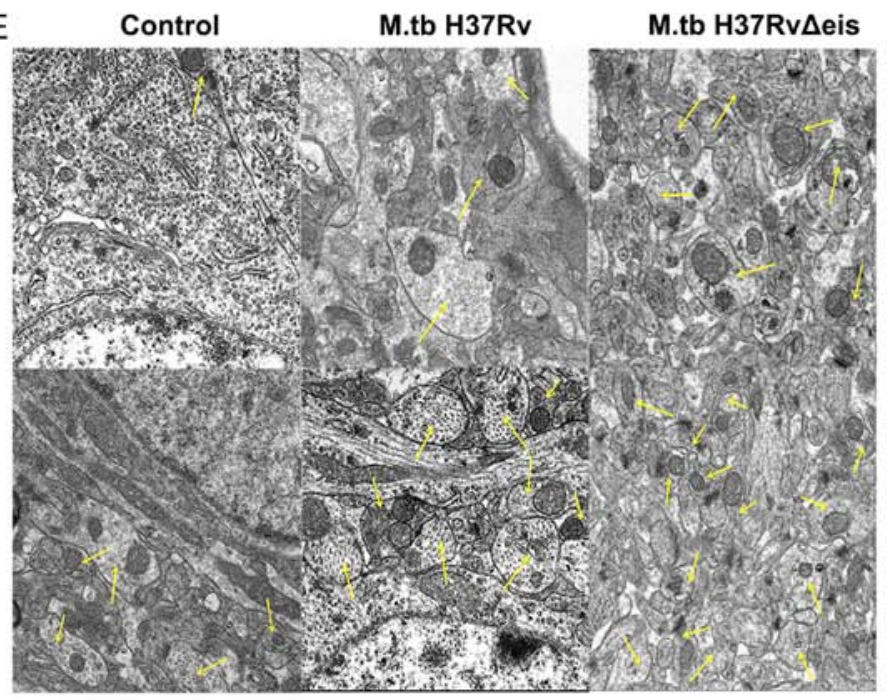

TNF- $\alpha$

M.tb H37Rv+TNF- $\alpha$ M.tb H37Rv $\Delta$ eis+TNF- $\alpha$

Figure 3. TNF- $\alpha$ enhances the autophagic activity of osteoclasts infected with M.tb. (A) Levels of TNF- $\alpha$ in control, M.tb H37Rv and M.tb H37Rv $\Delta$ eis groups mRNA expression levels of (B) Atg7 and (C) Beclin1 in osteoclasts infected with M.tb untreated or treated with TNF- $\alpha$. (D) Protein expression levels of Atg7, Beclin1 and LC3-II/I in osteoclasts infected with M.tb untreated or treated with TNF- $\alpha$. (E) Autophagosomes in osteoclasts infected with M.tb untreated or treated with TNF- $\alpha$ were observed by transmission electron microscopy (magnification, $\mathrm{x} 5,000$ ). Yellow arrows indicate autophagosomes. Scale bars, $1 \mu \mathrm{m}$. Data are presented as the mean $\pm \mathrm{SD} .{ }^{*} \mathrm{P}<0.05$ and ${ }^{* *} \mathrm{P}<0.01$. M.tb H37Rv $\Delta$ eis, inhibition of autophagy gene knockout type M.tb H37Rv; M.tbH37Rv wild type M.tb H37Rv; TNF- $\alpha$, tumor necrosis factor- $\alpha$; Atg7, autophagy-related protein 7; LC-3, microtubule-associated proteins 1A/1B light chain 3A; M.tb, Mycobacterium tuberculosis.

H37Rv-infected samples, whereas Bcl-2 mRNA levels significantly increased (Fig. 2D-F). Similarly, M.tb H37Rv $\Delta$ eis infection decreased the expression of cleaved-caspase 3 and Bax protein levels and increased the expression of anti-apoptotic Bcl-2 protein levels compared with controls and H37Rv-infected samples (Fig. 2G). In addition, attenuated translocation of mitochondrial cytochrome $\mathrm{c}$ to the cytoplasm was detected by western blot analysis (Fig. 2H).
TUNEL analysis further confirmed that autophagy reduced osteoclast apoptosis (Fig. 2I).

TNF- $\alpha$ enhances autophagic activity of osteoclasts infected with M.tb. TNF- $\alpha$ plays an important role in the development of tuberculosis (17). ELISA was performed to detect the secretion of TNF- $\alpha$ from osteoclasts infected with M.tb. As shown in Fig. 3A, M.tb infection significantly increased the 
levels of TNF- $\alpha$ compared with controls. Subsequently, the effect of TNF- $\alpha$ on osteoclasts infected with M.tb was tested. Osteoclasts infected with $M . t b$ were treated with TNF- $\alpha$ (40 ng/ml) for $24 \mathrm{~h}$. Untreated osteoclasts infected with M.tb were used as controls. RT-qPCR, western blotting and transmission electron microscopy were performed to determine autophagy activation. The results demonstrated that mRNA levels of Atg7 and Beclin1 were significantly upregulated in osteoclasts infected with M.tb and treated with TNF- $\alpha$ compared with corresponding cells that were not treated with TNF- $\alpha$ (Fig. 3B and C). Western blotting results showed that protein expression levels of Atg7, Beclin1 and LC3-II/I increased following TNF- $\alpha$ treatment of osteoblasts infected with M.tb (Fig. 3D). Furthermore, increased accumulation of autophagosomes was observed in infected osteoclasts treated with TNF- $\alpha$ as indicated by the arrows (Fig. 3E). The results indicated that TNF- $\alpha$ enhanced the autophagy of osteoclasts infected with M.tb.

TNF- $\alpha$ inhibits apoptosis of osteoclasts infected with M.tb by activating autophagy. To understand whether TNF- $\alpha$ affects the apoptosis of osteoclasts infected with M.tb, M.tb H37Rv $\Delta$ eis or M.tb H37Rv was transfected into human osteoclasts before the cells were stimulated with TNF- $\alpha$. Following osteoclast treatment with TNF- $\alpha$ (40 ng/ml) for $24 \mathrm{~h}$, mRNA and protein expression of apoptosis-associated factors were quantified by RT-qPCR and western blotting. The mRNA and protein expression of pro-apoptotic factors (cleaved-caspase 3 and Bax) in osteoclasts infected with M.tb significantly decreased after treatment with TNF- $\alpha$, whereas the mRNA and protein expression of anti-apoptotic $\mathrm{Bcl}-2$ was significantly increased compared with the respective TNF- $\alpha$-untreated groups (Fig. 4A-D). In addition, western blot analysis detected attenuated translocation of mitochondrial cytochrome $\mathrm{c}$ in TNF- $\alpha$-treated groups when compared with corresponding TNF- $\alpha$-untreated groups (Fig. 4E). Additionally, TUNEL staining showed that TNF- $\alpha$ treatment decreased the apoptosis of osteoclasts infected with M.tb (Fig. 4F).

To investigate the beneficial effects of TNF- $\alpha$ on the autophagy of osteoclasts infected with M.tb, cells were pre-treated with the autophagy inhibitor 3-MA before TNF- $\alpha$ administration. As shown in Fig. S1,3-MA treatment decreased the protein levels of Atg7, Beclin1 and LC3-II/I compared with the corresponding 3-MA-untreated groups. Pre-treatment with 3-MA significantly attenuated the effects of TNF- $\alpha$ on the expression of apoptosis-related factors compared with the respective 3-MA-untreated groups (Fig. 4G-K).

\section{Discussion}

Recent studies have shown that autophagy plays an important role in diverse biological and pathological processes, including cell proliferation, differentiation, apoptosis, and carcinogenesis; however, little is known about the role of autophagy in the development of osteoarticular tuberculosis (18-21). The present results demonstrated that the number of osteoclasts increased and autophagy was enhanced in the lesions of patients with osteoarticular tuberculosis compared with patients with OA. Activation of autophagy inhibited the apoptosis of osteoclasts infected with M.tb and increased the expression levels of TNF- $\alpha$. Furthermore, TNF- $\alpha$ enhanced the autophagic activity of osteoclasts infected with M.tb and inhibited cell apoptosis.

Osteoclasts are the only cells in the body that perform bone resorption and are closely related to normal bone metabolism and various diseases (22). The pathological changes in vertebrae destruction in spinal tuberculosis are closely related to abnormal activation and proliferation of osteoclasts caused by M.tb (23). The synergistic effect of osteoclasts and osteoblasts is crucial in bone formation and development. Once the balance between osteoclasts and osteoblasts is disrupted, abnormal bone diseases such as bone and joint tuberculosis can occur (24). Autophagy plays an important role in the formation, apoptosis and function of osteoclasts as an important mechanism of action to maintain cell homeostasis (25). Both osteoclast formation and bone resorption require the involvement of Atg5, Atg7, Atg4B and LC3. For example, Atg5 and Atg7 promote bone resorption in vivo and in vitro, mainly through the actin loop of osteoclasts as a target for lysozymes (26). Atg5, Atg7, Atg4B and LC3 are important components and regulatory molecules in autophagy, suggesting an important role for autophagy in osteoclast formation (19). LC3 is also involved in osteoclast differentiation, which is modified by Atg4B to block the absorption activity and expression of cathepsin $\mathrm{K}$ and inhibit the degradation of collagen in the bone matrix (27). The present results showed that tuberculosis infection induced autophagy, which inhibited the apoptosis of osteoclasts, further demonstrating a key role of autophagy in maintaining the osteoclast steady state.

TNF- $\alpha$ can promote granuloma formation, envelop tubercle bacillus, limit pathogen spread and promote pathological changes caused by tuberculosis (28). High levels of TNF- $\alpha$ were detected in the serum and cerebrospinal fluid of patients with spinal tuberculosis (29). TNF- $\alpha$, a pro-inflammatory factor, is key in the immunopathological response of tuberculosis. TNF- $\alpha$ can inhibit M.t $b$ infection and accelerate bone destruction. It is closely related to the occurrence, development, severity, therapeutic effect and prognosis of the disease (30). Additionally, Mattos et al (31) reported that the levels of TNF- $\alpha$ were significantly lower after tuberculosis chemotherapy than before, demonstrating that TNF- $\alpha$ may play an important role in osteoarticular tuberculosis. Once $M . t b$ enters the bone through the blood or lymphatic system, it activates mononuclear macrophages around the lesion to produce a large amount of TNF- $\alpha$, which directly acts on osteoclast precursor cells in the bone marrow to form a large number of osteoclasts, causing bone destruction (32). Kobayashi et al (33) reported that TNF- $\alpha$ directly induced osteoclast precursor cells (mononuclear macrophages) in the bone marrow to differentiate into osteoclasts, which does not depend on any other route. TNF- $\alpha$ was reported to directly induce osteoclast formation in the bone marrow, as well as induce osteoblast apoptosis, reduce the proliferation and differentiation of osteoblasts, inhibit new bone formation and aggravate bone destruction; thus, TNF- $\alpha$ inhibitors are effective for reducing bone loss $(34,35)$. Autophagy is activated by the proinflammatory cytokine TNF- $\alpha$ in the osteoclasts of patients with rheumatoid arthritis $(13,36)$. The present study showed that TNF- $\alpha$ enhanced autophagic activity and inhibited apoptosis of osteoclasts infected with M.tb. 


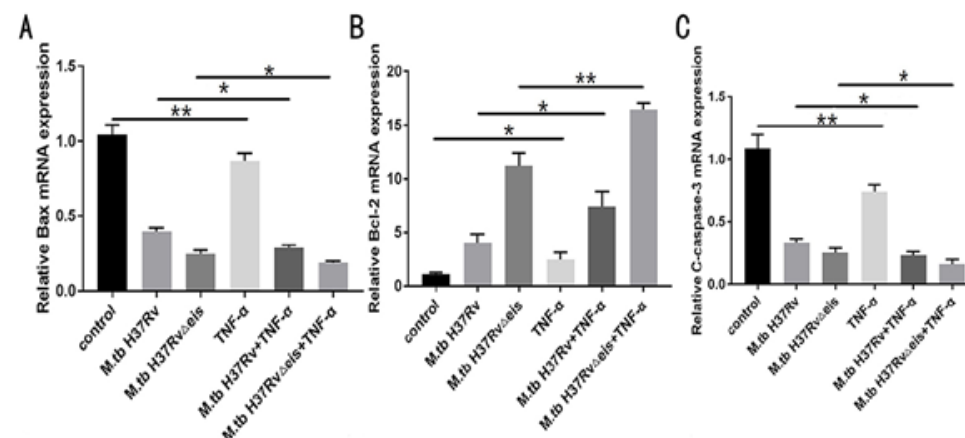

D
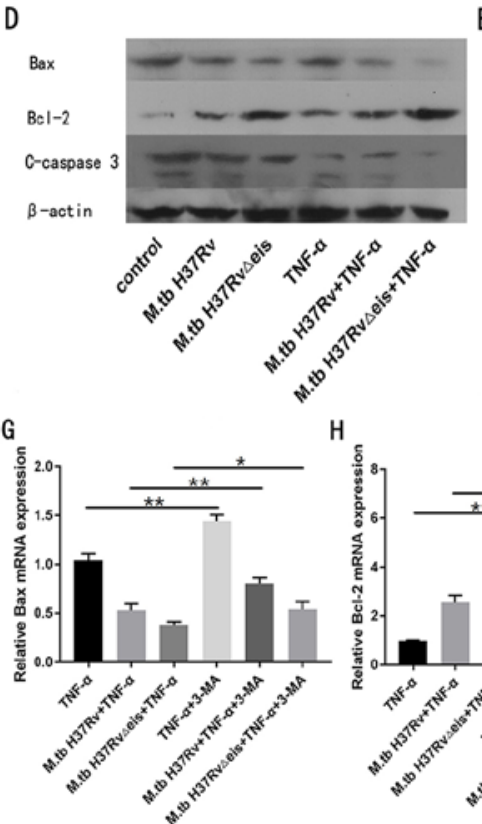

$J$

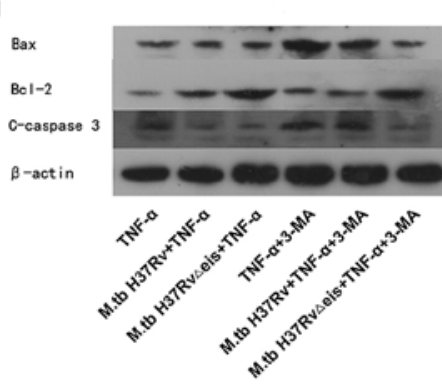

$\mathrm{E}$

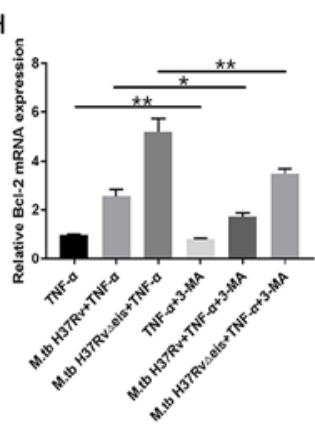

K

Cyto. Cyt-c$$
\text { . }
$$

VDAC
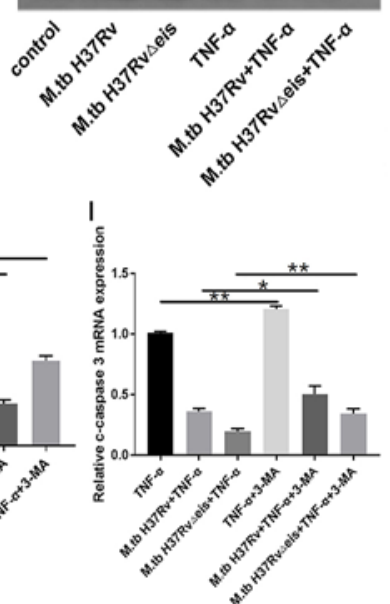

K

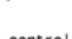

control

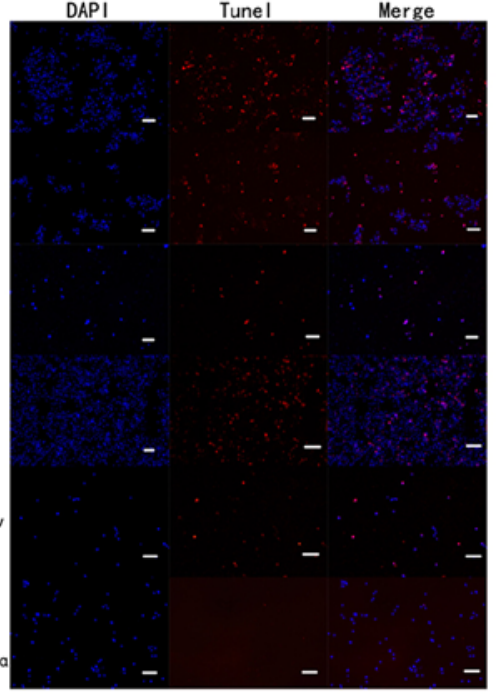

Figure 4. TNF- $\alpha$ inhibits apoptosis of osteoclasts infected with M.tb by activating autophagy. mRNA expression levels of (A) Bax, (B) Bcl-2 and (C) cleavedcaspase 3 in osteoclasts infected with M.tb untreated or treated with TNF- $\alpha$. (D) Protein levels of Bax, Bcl-2, and cleaved-caspase 3 in osteoclasts infected with M.tb untreated or treated with TNF- $\alpha$. (E) Western blotting assay of the levels of Cyt-c in cytoplasmic and mitochondrial extracts. (F) TUNEL assay was performed in osteoclasts infected with M.tb untreated or treated with TNF- $\alpha$. (magnification, x200; scale bar, $50 \mu \mathrm{m})$. mRNA expression levels of $(\mathrm{G}) \mathrm{Bax},(\mathrm{H})$ Bcl-2 and (I) cleaved-caspase 3 in osteoclasts infected with M.tb treated with TNF- $\alpha$, and untreated or treated with 3-MA. (J) Protein levels of Bax, Bcl-2, and cleaved-caspase 3 in osteoclasts infected with M.tb treated with TNF- $\alpha$, and untreated or treated with 3-MA. (K) Western blotting assay of the levels of Cyt-c in cytoplasmic and mitochondrial fractions of osteoclasts infected with M.tb treated with TNF- $\alpha$, and untreated or treated with 3-MA. Data are presented as the mean \pm SD. ${ }^{*} \mathrm{P}<0.05$ and $* * \mathrm{P}<0.01$. M.tbH37Rv $\Delta$ eis, inhibition of autophagy gene knockout type Mycobacterium tuberculosis H37Rv; M.tbH37Rv wild type Mycobacterium tuberculosis H37Rv; TNF- $\alpha$, tumor necrosis factor- $\alpha$; Atg7, autophagy-related protein 7; 3-MA, 3-methyladenine; VDAC, voltage-dependent anion channel.

Autophagy induced osteoclast differentiation and apoptosis and stimulated osteoclast-mediated bone resorption in vitro, thereby highlighting autophagy as a novel mediator of TNF- $\alpha$ induced bone resorption (37).

However, the present study had certain limitations. The study preliminarily explored the relationship between TNF- $\alpha$ and autophagy and did not determine the mechanism of TNF- $\alpha$-activated autophagy. Subsequent studies should be performed on TNF- $\alpha$-activated autophagy signaling pathways.
The present study was limited to the cellular level, and no animal experiments were performed. The results of this experiment should be further verified in animal models.

In conclusion, to the best of our knowledge, this is the first study to show that autophagy is an important factor in maintaining the homeostasis of osteoclasts infected with M.tb. Additionally, TNF- $\alpha$ promoted the autophagy of mature osteoclasts infected with $M . t b$ and inhibited the apoptosis of mature osteoclasts. These findings reveal a 
new osteoarticular tuberculosis-activated cytokine network through which autophagy may regulate bone metabolism and play an important role in the pathogenesis of osteoarticular tuberculosis. These results provide a foundation for the development of new drugs for treating osteoarticular tuberculosis based on TNF- $\alpha$-mediated osteoclast autophagy.

\section{Acknowledgements}

The authors would like to thank Dr Shuwen Jin from the College of Acupuncture of Orthopedics, Hubei University of Chinese Medicine, Wuhan, China for providing technical assistance. The authors would like to thank Professor Fan Xionglin, School of Basic Medicine, Tongji Medical College, Huazhong University of Science and Technology, Wuhan, China for providing the bacteria strain used in the study.

\section{Funding}

The current study is supported by grants from the Research Project of Hubei Province Health and Family Planning Commission (grant no. WJ2017Z022), Research Projects of Traditional Chinese Medicine of Hubei Health Commission (grant no. ZY2019F025), Wuhan Health and Family Planning Scientific Research Fund (grant nos. WZ18Q05 and WX19Q11) and the Funded Research Project of Wuhan First Hospital (grant no. 2019Y01).

\section{Availability of data and materials}

The datasets used and/or analyzed during the present study are available from the corresponding author on reasonable request.

\section{Authors' contributions}

WL contributed to study conception and design, data collection and analysis and manuscript writing. JZ and FN contributed to study conception and design and in critical revision and review of the manuscript. FFP, ZWW, MH and XLZ analyzed the data and reviewed the manuscript. LY and PFT performed the experiments. PX and JF analyzed and interpreted the data and contributed to final manuscript preparation. All authors read and approved the final manuscript.

\section{Ethics approval and consent to participate}

The present study was approved by the Clinical Research Ethics Committee of Wuhan First Hospital, Tongji Medical College, Huazhong University of Science Technology. All patients or their parents provided informed consent.

\section{Patient consent for publication}

Patients provided consent for publication.

\section{Competing interests}

The authors declare that they have no competing interests.

\section{References}

1. Tiwari U, Ramachandran VG, Das S and Kumar S: Interleukin-3 and interleukin-17 do not play a dynamic role in the immunopathogenesis of osteoarticular tuberculosis. Indian J Tuberc 61: 142-147, 2014.

2. Kaufmann SH, Lange C, Rao M, Balaji KN, Lotze M, Schito M, Zumla AI and Maeurer M: Progress in tuberculosis vaccine development and host-directed therapies-a state of the art review. Lancet Respir Med 2: 301-320, 2014.

3. Agarwal A: Acute suppurative presentation of osteoarticular tuberculosis in children. Indian J Tuberc 58: 66-71, 2011.

4. Hu P, Zhang H, Fleming J, Zhu G, Zhang S, Wang Y, Liu F, Yi S, Chen Z, Chen Z, et al: Retrospective analysis of false-positive and disputed rifampin resistance Xpert MTB/RIF assay results in clinical samples from a referral hospital in hunan, China. J Clin Microbiol 57: pii: e01707-e01718, 2019.

5. Wu W, Lyu J, Cheng P, Cheng Y, Zhang Z, Li L, Zheng Y and $\mathrm{Xu}$ J: Improvement in clinical outcome and infection control using molecular diagnostic techniques for early detection of MDR tuberculous spondylitis: A multicenter retrospective study. Emerg Microbes Infect 6: e97, 2017.

6. Trébucq A, Decroo T, Van Deun A, Piubello A, Chiang CY, Koura KG and Schwoebel V: Short-course regimen for multidrug-resistant tuberculosis: A decade of evidence. J Clin Med 9: pii: E55, 2019.

7. Zhou Y, Tan CY, Mo ZJ, Gao QL, He D, Li J, Huang RF, Li YB, Guo CF, Guo Q, et al: Polymorphisms in the SP110 and TNF- $\alpha$ gene and susceptibility to pulmonary and spinal tuberculosis among southern chinese population. Dis Markers 2017: 4590235, 2017.

8. Meghji S, White PA, Nair SP, Reddi K, Heron K, Henderson B, Zaliani A, Fossati G, Mascagni P, Hunt JF, et al: Mycobacterium tuberculosis chaperonin 10 stimulates bone resorption: A potential contributory factor in Pott's disease. J Exp Med 186: 1241-1246, 1997.

9. Liu X, Jia W, Wang H, Wang Y, Ma J, Wang H, Zhou X and Li G: Establishment of a rabbit model of spinal tuberculosis using Mycobacterium tuberculosis strain H37Rv. Jpn J Infect Dis 68: 89-97, 2015.

10. Lawlor C, O'Connor G, O'Leary S, Gallagher PJ, Cryan SA, Keane J and O'Sullivan MP: Treatment of Mycobacterium tuberculosis-infected macrophages with Poly(Lactic-Co-Glycolic Acid) microparticles drives $\mathrm{NF} \kappa \mathrm{B}$ and autophagy dependent bacillary killing. PLoS One 11: e0149167, 2016.

11. Tateosian NL, Pellegrini JM, Amiano NO, Rolandelli A, Casco N, Palmero DJ, Colombo MI and García VE: IL17A augments autophagy in Mycobacterium tuberculosis-infected monocytes from patients with active tuberculosis in association with the severity of the disease. Autophagy 13: 1191-1204, 2017.

12. Ozeki N, Mogi M, Hase N, Hiyama T, Yamaguchi $\mathrm{H}$, Kawai R, Matsumoto T and Nakata K: Bone morphogenetic protein-induced cell differentiation involves Atg7 and Wnt16 sequentially in human stem cell-derived osteoblastic cells. Exp Cell Res 347: 24-41, 2016.

13. Lin NY, Beyer C, Giessl A, Kireva T, Scholtysek C, Uderhardt S, Munoz LE, Dees C, Distler A, Wirtz S, et al: Autophagy regulates $\mathrm{TNF} \alpha$-mediated joint destruction in experimental arthritis. Ann Rheum Dis 72: 761-768, 2013.

14. Espert L, Beaumelle B and Vergne I: Autophagy in Mycobacterium tuberculosis and HIV infections. Front Cell Infect Microbio 5: 49, 2015.

15. Gutierrez MG, Master SS, Singh SB, Taylor GA, Colombo MI and Deretic V: Autophagy is a defense mechanism inhibiting BCG and Mycobacterium tuberculosis survival in infected macrophages. Cell 119: 753-766, 2004.

16. Livak KJ and Schmittgen TD: Analysis of relative gene expression data using real-time quantitative PCR and the 2(-Delta Delta C(T)) method. Methods 25: 402-408, 2001.

17. Aydin V, Akici A, Isli F, Aksoy M, Aydin M and Gursoz H: Relative risk of tuberculosis in patients with rheumatic diseases managed with anti-tumour necrosis factor-alpha therapy: A nationwide cohort study. J Clin Pharm The 44: 553-560, 2019.

18. Guo C, Wang L, Zhao Y, Jiang B, Luo J and Shi D: BOS-93, a novel bromophenol derivative, induces apoptosis and autophagy in human A549 lung cancer cells via PI3K/Akt/mTOR and MAPK signaling pathway. Exp Ther Med 17: 3848-3858, 2019.

19. Arai A, Kim S, Goldshteyn V, Kim T, Park NH, Wang CY and Kim RH: Beclin1 modulates bone homeostasis by regulating osteoclast and chondrocyte differentiation. J Bone Miner Res 34: 1753-1766, 2019. 
20. Sabir N, Hussain T, Liao Y, Wang J, Song Y, Shahid M, Cheng G, Mangi MH, Yao J, Yang L, et al: Kallikrein 12 regulates innate resistance of murine macrophages against Mycobacterium bovis infection by modulating autophagy and apoptosis. Cells 8: pii: E415, 2019.

21. Trejo-Solís C, Serrano-Garcia N, Escamilla-Ramírez Á, Castillo-Rodríguez RA, Jimenez-Farfan D, Palencia G, Calvillo M, Alvarez-Lemus MA, Flores-Nájera A, Cruz-Salgado A and Sotelo J: Autophagic and Apoptotic pathways as targets for chemotherapy in glioblastoma. Int J Mol Sci 19: pii: E3773, 2018.

22. Park YE, Musson DS, Naot D and Cornish J: Cell-cell communication in bone development and whole-body homeostasis and pharmacological avenues for bone disorders. Curr Opin Pharmacol 34: 21-35, 2017.

23. Hoshino A, Hanada S, Yamada H, Mii S, Takahashi M, Mitarai S, Yamamoto K and Manome Y: Mycobacterium tuberculosis escapes from the phagosomes of infected human osteoclasts reprograms osteoclast development via dysregulation of cytokines and chemokines. Pathog Dis 70: 28-39, 2014.

24. Yi L, Li Z, Jiang H, Cao Z, Liu J and Zhang X: Gene modification of transforming growth factor $\beta$ (TGF- $\beta$ ) and interleukin 10 (IL-10) in suppressing Mt sonicate induced osteoclast formation and bone absorption. Med Sci Monit 24: 5200-5207, 2018.

25. Sun KT, Chen MY, Tu MG, Wang IK, Chang SS and Li CY: MicroRNA-20a regulates autophagy related protein-ATG16L1 in hypoxia-induced osteoclast differentiation. Bone 73: 145-153, 2015.

26. DeSelm CJ, Miller BC, Zou W, Beatty WL, van Meel E, Takahata Y, Klumperman J, Tooze SA, Teitelbaum SL and Virgin HW: Autophagy proteins regulate the secretory component of osteoclastic bone resorption. Dev Cell 21: 966-974, 2011.

27. Xing L, Xiu Y and Boyce BF: Osteoclast fusion and regulation by RANKL-dependent and independent factors. World J Orthop 3: 212-222, 2012

28. Mootoo A, Stylianou E, Arias MA and Reljic R: TNF-alpha in tuberculosis: A cytokine with a split personality. Inflamm Allergy Drug Targets 8: 53-62, 2009.

29. Chen H, Cheng C, Li M, Gao S, Li S and Sun H: Expression of TNF- $\alpha$, IFN- $\gamma$, TGF- $\beta$ and IL- 4 in the spinal tuberculous focus and its impact on the disease. Cell Biochem Biophys 70 : $1759-1764,2014$.
30. Patil T, Garg RK, Jain A, Goel MM, Malhotra HS, Verma R, Singh GP and Sharma PK: Serum and CSF cytokines and matrix metalloproteinases in spinal tuberculosis. Inflamm Res 64: 97-106, 2015

31. Mattos AM, Almeida Cde S, Franken KL, Alves CC, Abramo C, de Souza MA, L'Hotellier M, Alves MJ, Ferreira AP, Oliveira SC, et al: Increased IgG1, IFN-gamma, TNF-alpha and IL-6 responses to Mycobacterium tuberculosis antigens in patients with tuberculosis are lower after chemotherapy. Int Immunol 22: 775-782, 2010.

32. Zheng M, Shi S, Wei W, Zheng Q, Wang Y, Ying X and Lu D: Correlation between MBL2/CD14/TNF- $\alpha$ gene polymorphisms and susceptibility to spinal tuberculosis in Chinese population. Biosci Rep 38: pii: BSR20171140, 2018.

33. Kobayashi K, Takahashi N, Jimi E, Udagawa N, Takami M, Kotake S, Nakagawa N,Kinosaki M, Yamaguchi K, Shima N, et al: Tumor necrosis factor alpha stimulates osteoclast differentiation by a mechanism independent of the ODF/RANKL-RANK interaction. J Exp Med 191: 275-286, 2000.

34. Osta B, Benedetti G and Miossec P: Classical and paradoxical effects of TNF- $\alpha$ on bone homeostasis. Front Immunol 5: 48, 2014.

35. Kawai VK, Stein CM, Perrien DS and Griffin MR: Effects of anti-tumor necrosis factor $\alpha$ agents on bone. Curr Opin Rheumatol 24: 576-585, 2012.

36. Lin NY, Stefanica A and Distler JH: Autophagy: A key pathway of TNF-induced inflammatory bone loss. Autophagy 9: 1253-1255, 2013.

37. Tong X, Gu J, Song R, Wang D, Sun Z, Sui C, Zhang C, Liu X, Bian J and Liu Z: Osteoprotegerin inhibit osteoclast differentiation and bone resorption by enhancing autophagy via AMPK/mTOR/p70S6K signaling pathway in vitro. J Cell Biochem 2018, doi: 10.1002/jcb.27468. [Epub ahead of print]

This work is licensed under a Creative Commons Attribution-NonCommercial-NoDerivatives 4.0 International (CC BY-NC-ND 4.0) License. 